\title{
Henoch-Schönlein Purpura Nephritis
}

National Cancer Institute

\section{Source}

National Cancer Institute. Henoch-Schönlein Purpura Nephritis. NCI Thesaurus. Code C123181.

Glomerulonephritis in the context of Henoch-Schönlein purpura. 